\title{
LA-UR-13-23153
}

Approved for public release; distribution is unlimited.

Title:

Author(s):

Intended for:

\section{CBTF Code Workshop}

Mason, Michael A.

Montoya, David R.

Release for future users of CBTF.

Web

Issued:

2013-05-01

Disclaimer:

Los Alamos National Laboratory, an affirmative action/equal opportunity employer,is operated by the Los Alamos National

Security, LLC for the National NuclearSecurity Administration of the U.S. Department of Energy under contract DE-AC52-06NA25396. By approving this article, the publisher recognizes that the U.S. Government retains nonexclusive, royalty-free license to publish or reproduce the published form of this contribution, or to allow others to do so, for U.S. Government purposes.

Los Alamos National Laboratory requests that the publisher identify this article as work performed under the auspices of the

U.S. Departmentof Energy. Los Alamos National Laboratory strongly supports academic freedom and a researcher's right to publish; as an institution, however, the Laboratory does not endorse the viewpoint of a publication or guarantee its technical correctness. 


\section{CBTF Code Workshop}

Component Based Tool Framework

Code Workshop

Mike Mason

Dave Montoya

LANL, HPC-3
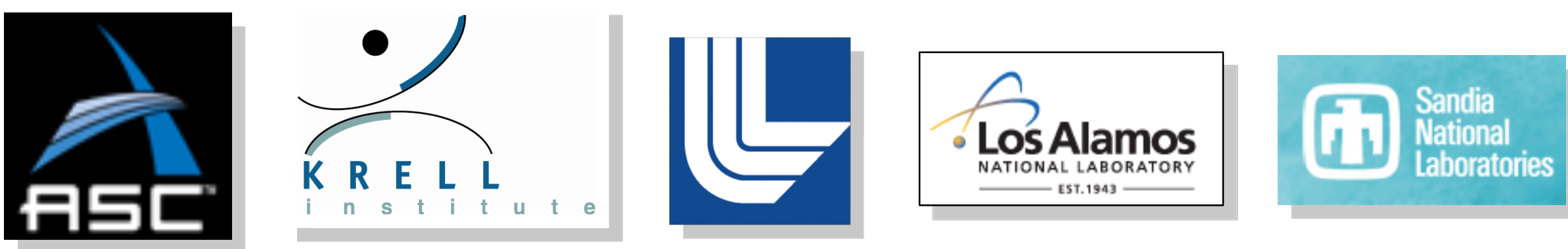

Open | SpeedShop"' 


\section{* What is CBTF?}

$>$ A Framework for writing Tools that are Based on Components.

$*$ Benefits of CBTF

$>$ CBTF is flexible and general enough to be used for any tool that needs to "do something" on a large number of nodes and filter or collect the results.

$>$ Components are reusable and easily added to new tools.

$>$ With a large component repository new tools can be written quickly with little code.

$>$ Tools automatically adjust to new topologies.

$>$ Tools are inherently scalable.

* Originally made for Performance Analysis with Open|SpeedShop

$>$ We have alternative uses like sysadmin or monitoring tools. 


\section{Components}

* Reusable objects with 0-N inputs and 0-M outputs.

* Uses the dataflow programming model where each component is a black box that takes some input, performs a task then produces some output.

* Designed to be connected together

$>$ Connections defined in $\mathrm{C}++$ or XML file.

* Components are written in $\mathrm{C}++$

$>$ Components can do anything your $\mathrm{C}++$ code can do.

$>$ Run system commands, open files, do calculations. 
* Three Networks where components can be connected

> Frontend, Backend, multiple Filer levels

$>$ Every level is homogeneous

* Each Network is also a component and therefore has some number of inputs and outputs.

* Any component can be run on any level, but logically

$>$ Frontend

- Interact with or Display info to the user

$>$ Filter

- Filter or Aggregate info from below

- Make decisions about what is sent up or

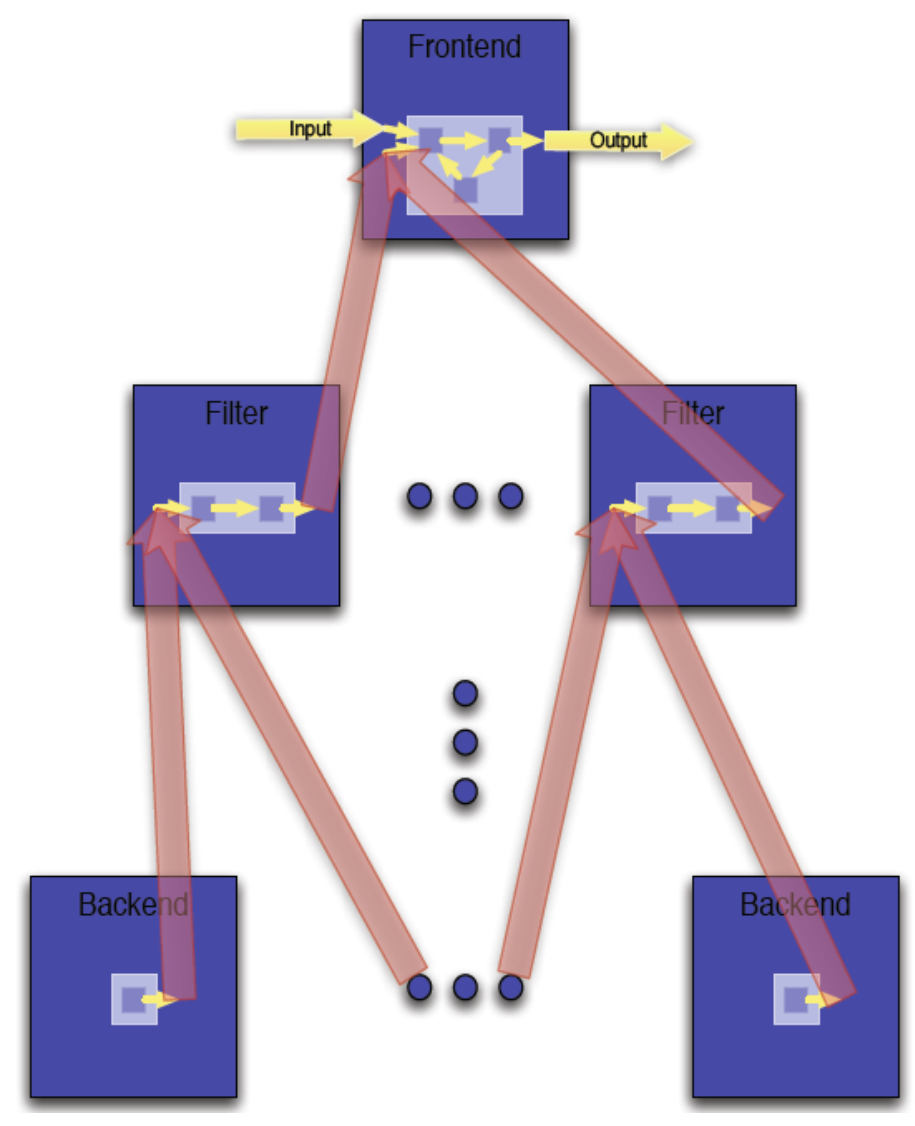
down the tree

$>$ Backend

- Real work of the tool 
- CBTF uses another mechanism to transport all of its communications.

* Right now that mechanism is MRNet

$>$ Multicast/Reduction Network

> Tree structure lends itself to improved scalability

* CBTF views MRNet as just another component.

$>$ In the future it can be easily swapped with some other transport mechanism.

> It can be used within a component network already running on MRNet allowing for some control over the tree structure at runtime.

> We do break some abstraction to use features of MRNet so right now it really cannot be swapped out easily.

\section{* Topology}

> You must define a tree based topology with one root (Frontend) node, zero or more levels of communication process (Filter) nodes and some number of leaf (Backend) nodes.

- Normal mode (Backend Create)

> Both MRNet and CBTF components are started on the Backend automatically.

* Lightweight mode (Backend Attach)

$>$ The MRNet tree is created but not on the Backend. An application is run separately on the Backend that attaches itself to the MRNet tree. 
* There are 3 basic files needed for a CBTF Tool

$>$ Tool file

- $\mathrm{C}++$

- Load the needed files, sets up MRNet, sends and receives data from the CBTF network.

$>$ Component (Plugin) file

- Generally C++ but can also be an XML network of other components

- Defines the components and filters, their inputs/outputs and what they do with the data.

$>$ XML file

- XML

- Defines the connections between the components and the streams sent between networks.

* We will look at the code for a tool called stack. 


\section{* Stack trace grouping (cbtf/tools/contrib/stack)}

$>$ Run gstack on each pid for a parallel application and group similar stack traces together.

mmason@rra012a: /mpi -

\section{(6) mmason@rr-dev-fe:stack - ssh - 80×49}

[mmasonerr-dev-fe stack]\$./stack mpi_hang
tput: No value for $\$ T E R M$ and no $-T$ specified

tput: No value for \$TERM and no -T specified

7236241.671897: FROMCHILD(rra012a: 0 ) (0x40cc0950)
omChildren() - stream 1073741825 lookup failed

7236241.672057: FROMCHILD(rra012a:0)(0x40cc0950)

rom 7236241.672234: FROMCHILD(rra012a: 0) (0x40cc0950

(2650): PeerNode.C[264] recv_thread_ma in() proc_PacketsFromChildren() failed

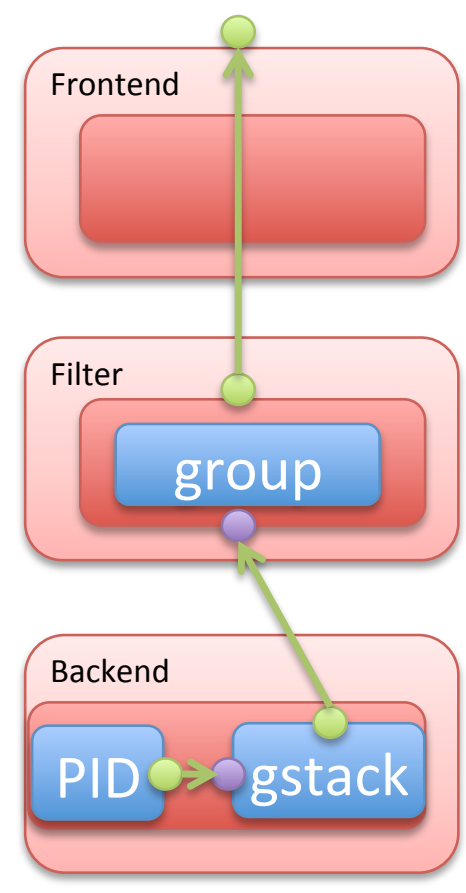
Thread 3 (Thm g (

[mmasonerra012a /mpi]\$ mpirun -np 4 ./mpi_hang \#a \#\# in select \& $\mathrm{Trom} /$ lib64/libc.so.6

Reported: 1(1) daemons 4(4) procs

rank 0 started my_rank $=0$

$\wedge \mathrm{Z}$

Suspended

[mmasonerra012a $\sim \mathrm{mpi}$ ] ps -u mmason PID TTY $8214 \mathrm{pts} / 0$

8222 pts $/ 0$

8275 pts $/ 0$

$8277 \mathrm{pts} / 0$

8278 pts $/ 0$

8279 pts $/ 0$

$8280 \mathrm{pts} / 0$

$8290 \mathrm{pts} / 0$

[mmasonerra012a $/ \mathrm{mpi}] \$$ ps

\#1 \#\#\# in service_thread_start ()

\#2) \#\#\# in start_thread () from /lib64/libpthread.so.0

\#3 \#\#\# in clone () from / lib64/libc.so.6

Thread 2 (Thread \#\#\# (LWP \#\#\#)):

\#0 \#\# in poll () from /lib64/libc.so.6

\#1 \#\#\# in btl_openib_async_thread ()

\#2 \#\#\# in start_thread () from /lib64/libpthread.so.0

$00: 00: 00 \mathrm{tcsh}$

$00: 00: 00$ pbs_mom

$00: 00: 00$ mpirun

$00: 00: 02$ mpi_hang

00:00:00 mpi hang

00:00:02 mpi_hang

$00: 00: 00$ mpi_hang

$00: 00: 00 \mathrm{ps}$

\#3 \#\#\# in clone () from /lib64/libc.so.6

Thread 1 (Thread \#\#\# (LWP \#\#\#)):

\#■ \#\#\# in mca_btl_sm_component_progress ()

\#1 \#\#\# in opal_progress ()

\#2 \#\# in opal_progress () in

\#2 \#\#\# in ompi_request_default_wait_alt ()

4 \#\#\# in ompi_coll_tuned_barrier_intra_recursivedoubling ()

5 \#\#\# in PMPI_Barrier ()

\#6 H\#\# in main ()

rra012a.rr. lanl.gov(8278) rra012a.rr.lanl.gov(8280)

Thread 3 (Thread \#\#\# (LWr \#\#\#)).

\#0 \#\#\# in select () from/lib64/libc.so.6

\#1 \#\#\# in service_thread_start ()

\#2 \#\#\# in start thread () from /lib64/libpthread.so.0

\#3 \#\#\# in clone () from /lib64/libc.so.6

Thread 2 (Thread \#\#\# (LWP \#\#)):

\#0 \#\#\# in poll () from/lib64/libc.so.6

\#1 \#\#\# in btl_openib_async_thread ()

\#2 \#\# in start_thread () from / lib64/l

\#3 \#\#\# in clone () from/lib64/libc.so.6

Thread 1 (Thread \#\#\# (LWP \#\#\#)):

\#0 \#\#\# in poll () from /lib64/libc.so.6

\#1 \#\#\# in poll_dispatch ()

\#2 \#\#\# in opal_event_base_loop ()

\#3 \#\# in opal_progress ()

\#4 \#\#\# in barrier ()

\#5 \#\# in ompi_mpi_finalize ()

\#6 \#\# in main () 
* Backend

$>\operatorname{llogin}-\mathrm{nn} 1$

$>$ /users/mmason/mpi

> mpirun-np 4 ./mpi_hang

* Frontend

$>\sim$ /.cbtf/cbtf_topology

- rr-dev-fe.lanl.gov:0 =>

- rra008a.rr.lanl.gov:0 ;

$>$ /usr/projects/packages/mmason/opt/cbtf-rr/share/ KrellInstitute/contrib/bin

$>$./stack mpi_hang

* One filter component performing cluster analysis on the output grouping together similar stack traces from gstack output. 
* C++

* Include

$>$ Boost

$>$ Krell Institute files

$>$ MRNet

$>$ Anything else you need string, vector ...

* Main

$>$ Register XML

$>$ Setup MRNet

- topology_file

$>$ Necessary for initial input/output

$>$ Does basic CBTF network connections

* This file is usually pretty simple. It may be possible to make a generic tool loader. 


\section{* Include boost, Krell and anything else}

\#include <boost/shared_ptr.hpp>

\#include <boost/program_options.hpp >

\#include <sys/param.h>

\#include <mrnet/MRNet.h>

\#include <typeinfo> \#include <KrellInstitute/CBTF/BoostExts.hpp > \#include <KrellInstitute/CBTF/Component.hpp > \#include <KrellInstitute/CBTF/Type.hpp> \#include <KrellInstitute/CBTF/ValueSink.hpp> \#include $\langle$ KrellInstitute/CBTF/ValueSource.hpp $>$ $\#$ \#include <KrellInstitute/CBTF/Version.hpp $>$ \#include <KrellInstitute/CBTF/XML.hpp >

\begin{abstract}
\#include <iostream>
\#include <map >

\#include <set>

\#include <stdexcept>

\#include <string $>$

\#include <stdio.h>

\#include <sstream $>$

\#include <unistd.h>

\#include < vector $>$
\end{abstract}

\section{* Main}

int main(int argc, char *argv[ ])

$\{\ldots$

registerXML(boost::filesystem::path(XMLDIR) / "stack.xml");

Component::registerPlugin(boost::filesystem::path(LIBDIR) / "KrellInstitute/

CBTF/BasicMRNetLaunchers.so" );

Component::Instance launcher $=$ Component::instantiate(

Type("BasicMRNetLauncherUsingBackendCreate"));

// Setup Network

Component::Instance network;

network = Component::instantiate(Type("stackNetwork"));

Component::connect(launcher, "Network", network, "Network");

// Setup Topology file

boost::shared_ptr $<$ ValueSource<boost::filesystem::path $>>$ topology_file $=$

ValueSource<boost::filesystem::path>::instantiate();

Component::Instance topology_file_component =

boost::reinterpret_pointer_cast<Component>(topology_file);

Component::connect(topology_file_component, "value", launcher,

"TopologyFile");
// Create the input

boost::shared_ptr<ValueSource<std::string $>>$ input_value $=$

ValueSource<std::string $>$ ::instantiate();

Component::Instance input_value_component =

boost::reinterpret_pointer_cast<Component>(input_value);

Component::connect(input_value_component, "value", network, "in");

// Create the output

boost::shared_ptr<ValueSink<std::vector $<$ std::string $>>>$ output_value = ValueSink<std::vector<std::string> >::instantiate();

Component::Instance output_value_component =

boost::reinterpret_pointer_cast<Component>(output_value);

Component::connect(network, "out", output_value_component, "value");

// Start mrnet network

default_topology += "/.cbtf/cbtf_topology";

*topology_file = default_topology;

// Send the app name down the mrnet tree

*input_value $=\operatorname{argv}[1]$

// Wait for output

std::vector<std::string > output = *output_value; 


\section{Component (Plugin) file}

* C++ Classes (or Networks of such classes)

$>$ You change very little

- Name of class

- Fill in the input handlers you add

- Must declare class as a CBTF component using the KRELL_INSTITUTE_CBTF_REGISTER_FACTORY_FUNCTION(class) macro

\section{* Input/Output}

$>$ declarelnput<InputType>("InputName", FunctionToCall);

$>$ declareOutput<OutputType>("OutputName");

\section{* inHandler}

$>$ Normal C++ function, do anything you want with the input

$>$ emitOutput<OutputType>("OutputName", output );

* Note that emitOutput is blocking, it doesn't return until the component that took in the output returns.

$>$ They are working on an asynchronous mode that doesn't block

* MRNet info

> include KrellInstitute/CBTF/Impl/MRNet.hpp

$>$ struct KrellInstitute::CBTF::Impl::TheTopologyInfo 


\section{Component (Plugin) file Code}

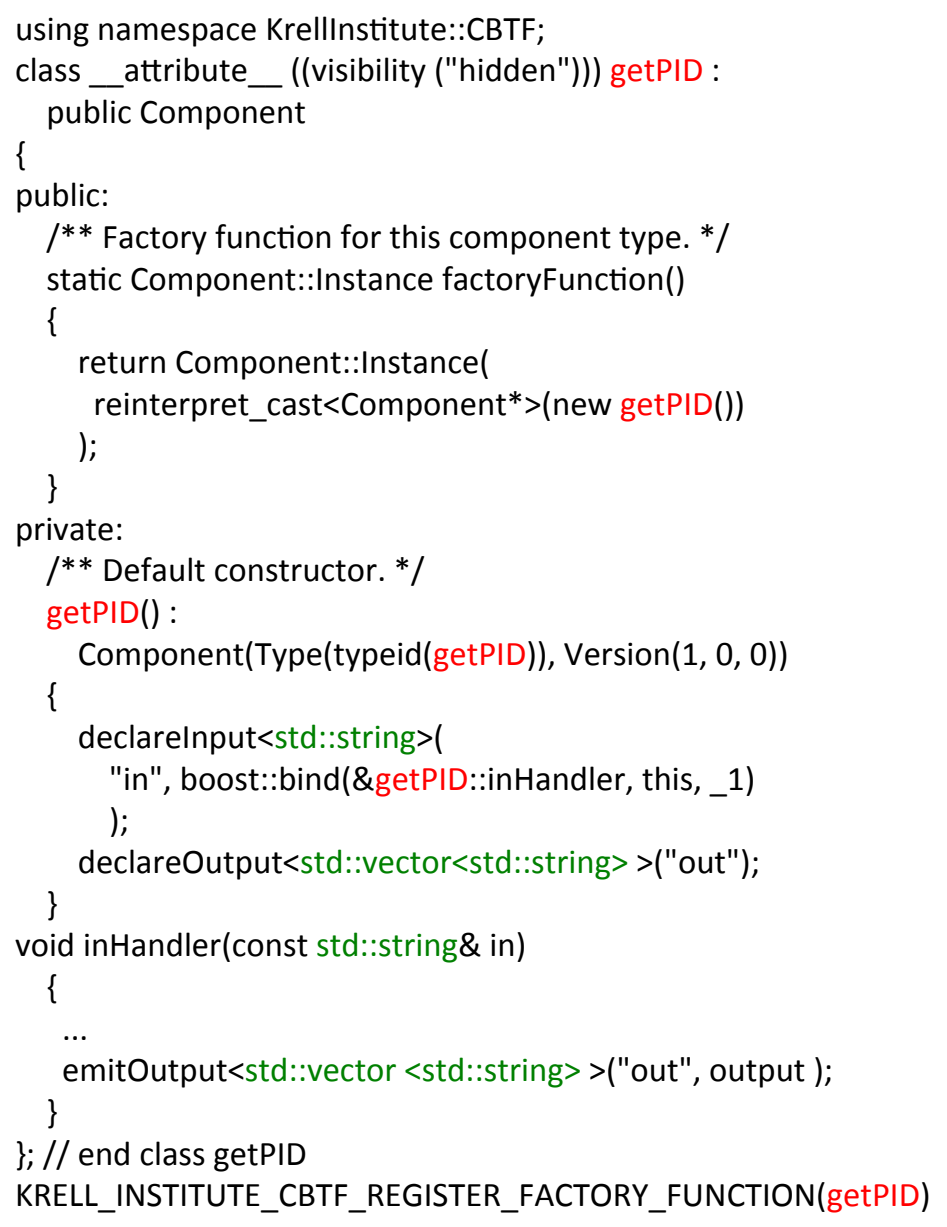


* XML file

> Schema cbtf/framework/libcbtf-xml/Network.xsd

* Type

$>$ Loaded as the Network Component in the $\mathrm{C}++$ Tool file

* Input/Output for the system

$>$ Use in the $\mathrm{C}++$ Tool file

* Networks for each Node type: Frontend, Filter, Backend

$>$ SearchPath (to the component repository)

$>$ Plugin (the compiled $\mathrm{C}++$ component file)

$>$ Component

$>$ Inputs/Outputs for the network

$>$ Connection

$>$ Streams (defined outside the network)

- IncomingDownstream, OutgoingUpstream (for Backend) 
<MRNet xmlns="http://www.krellinst.org/CBTF/MRNet.xsd"> $<$ Type>stackNetwork</Type>

$<$ Version $>1.0 .0</$ Version $>$

$<$ Input $>$

$<$ Name $>$ in $<$ /Name $>$

$<$ To $><$ Input $>$ system_in $</$ Input $></$ To $>$

$</$ Input $>$

$<$ Output>

$<$ Name $>$ out $<$ /Name $>$

$<$ From $><$ Output $>$ system_out $</$ Output $></$ From $>$ $</$ Output $>$

$<$ Frontend $>$

$<$ Network>

$<$ Type $>$ stackNetwork_Frontend</Type $>$

$<$ Version $>1.0 .0<$ /Version $>$

$\cdots$
$<$ Filter $>$

$<$ Depth $><$ RootRelative offset="0"/ $></$ Depth $>$

$<$ Network $>$

$<$ Type $>$ stackNetwork_Filter</Type $>$

$<$ Version $>1.0 .0<$ /Version $>$

$<$ SearchPath>/usr/projects/packages/mmason/opt/cbtf-

$\mathrm{rr} /$ share/KrellInstitute/contrib/lib $</$ SearchPath $>$

$<$ Plugin $>$ mrnetPlugin.so</Plugin $>$

$<$ Plugin $>$ stackPlugin.so</Plugin $>$

$<$ Component>

$<$ Name $>$ groupStackFilter $<$ Name $>$

$<$ Type $>$ groupStack $</$ Type $>$

$</$ Component $>$

$</$ Network $>$

$<$ !-- stream from backend -->

$<$ IncomingUpstream>

$<$ Name $>$ UpwardStream $<$ Name $>$

$<$ To $><$ Input $>$ Filter_In $</$ Input $></$ To $>$

$</$ IncomingUpstream $>$

$<$ !-- stream to frontend -->

$<$ OutgoingUpstream>

$<$ Name $>$ UpwardStream $<$ Name $>$

$<$ From $><$ Output $>$ Filter_Output $<$ /Output $><$ /From $>$

$</$ OutgoingUpstream $>$

$</$ Filter $>$
$<$ Backend $>$

$<$ Network>

$<$ Type $>$ TestNetwork_Backend</Type>

$<$ Version $>1.0 .0</$ Version $>$

$<$ SearchPath $>/$ usr/projects/packages/mmason

/opt/cbtf-rr/share/Krellinstitute/contrib/lib

$</$ SearchPath $>$

$<$ Plugin>mrnetPlugin.so</Plugin $>$

$<$ Plugin $>$ stackPlugin.so</Plugin $>$

$<$ Component $>$

$<$ Name $>$ getPID $</$ Name $>$

$<$ Type>getPID</Type >

$</$ Component $>$

...

$<$ Connection $>$

$<$ From $>$

$<$ Name $>$ getPID $<$ /Name $>$

$<$ Output $>$ out $<$ /Output $>$

$</$ From $>$

$<$ To>

$<$ Name $>$ getStack $<$ /Name $>$

$<$ Input $>$ in $</$ Input $>$

$</$ To $>$

$</$ Connection $>$ 
Building your Tool

* XXXXtool.tar.gz

$>$ Creates a tool template ready to compile from your home directory (it is a little out of date right now)

* ./bootstrap

* /configure

$>$ Look at the options in cbtf/tools/examples/config.log

* make

* make install 


\section{Installing CBTF}

\section{* cbtf_root}

$>$ Install first

$>$ Contains all of the things needed to build CBTF

- Boost, MRNet, etc...

$>$./install-cbtf --install-prefix <install-dir (opt/cbtf)> --build-all

Installed components status:

- binutils- not installed

- libelf- not installed

- libdwarf- 2012-01-10

- libunwind- 2012-01-10

- papi- 2012-01-10

- monitor- 2012-01-10

- dyninst- 2012-01-10

- mrnet- 2012-01-10

- CBTF- building from tarball not supported yet

- boost- 2012-01-10

- xerces-c- 2012-01-10

cbtf

$>$ Use the install command from above

\section{* Install Requires}

$>$ cmake $>=2.8$ that version is not on most clusters

$>$ Newer autotools

- ./install-cbtf --install-prefix <install-dir>/opt/autotools --build-autotools 


\section{Setup your environment for CBTF}

\section{* We use our own module file to setup CBTF}

set cbtf_root /usr/projects/packages/mmason/opt/cbtf-rr

$\begin{array}{lll}\text { setenv } & \text { CBTF_HOME } & \text { \$cbtf_root } \\ \text { setenv } & \text { CBTF_INSTALL_DIR } & \text { \$cbtf_root } \\ \text { setenv } & \text { CBTF_PREFIX } & \text { \$cbtf_root } \\ \text { setenv } & \text { CBTF_DOC_DIR } & \text { \$cbtf_root/share/doc/par_tion openmpi } \\ \text { setenv } & \text { CBTF_MPI_IMPLEMENTATION } & \text { ssh } \\ \text { setenv } & \text { XPLAT_RSH } & \text { ssh } \\ \text { setenv } & \text { XPLAT_RSHCOMMAND } & \\ & & \\ \text { prepend-path } & \text { PATH } & \text { \$cbtf_root/bin } \\ \text { prepend-path } & \text { MANPATH } & \text { \$cbtf_root/share/man }\end{array}$




\section{CBTF directory tree}

* cbtf

$>$ Tools

- contrib

- Our code that we add to the git repository

- examples/src

- daemonToolDemo

- pcsamp

$>$ Framework

- examples

- May be out of date

\section{* <install-dir>/opt/cbtf}

$>$ share/KrellInstitute/contrib

- bin lib xml

$>$ share/KrellInstitute/demos

- daemonToolDemo, pcsamp 
* Problems

$>$ CBTF is still being written

> Very dependent on MRNet

- Not easily changeable

- Must know about MRNet and how to use it

$>\mathrm{XML}$ file gets big and complicated fast

- At some point there will be a GUI viewer/builder

\section{* Summary}

> Provides a framework for writing scalable tools.

> Easily reuse and add components to tools.

> We can make simple example tools now

- Not ready for the real world yet 